\title{
Manifestações orais em pacientes portadores do diabetes mellitus: uma revisão narrativa
}

\author{
Oral manifestations in patients with diabetes mellitus: a narrative review
}

Manifestaciones orales en pacientes con diabetes mellitus: una revisión narrativa

Caroline Rodrigues Thomes ${ }^{1 *}$, Jonata Leal dos Santos ${ }^{2}$, Lara Victória Dittz de Abreu Costa ${ }^{1}$, David Wilkerson dos Santos Silva ${ }^{2}$, Elisama de Oliveira Mendes ${ }^{2}$, Wendel Chaves Carvalho ${ }^{3}$, Rafael Oliveira de Paula ${ }^{2}$, Antonio Weynisson Felix Santana ${ }^{4}$, Ernandes Aparecido Santos ${ }^{5}$, Alfredo Carlos Rodrigues Feitosa1.

\section{RESUMO}

Objetivo: Descrever as manifestações orais provenientes do diabetes mellitus. Revisão bibliográfica: $O$ diabetes mellitus é o distúrbio metabólico mais comum, causador de repercussões multiviscerais e classificado em tipo 1 e tipo 2. Quando descontrolado pode gerar uma série de complicações renais, oculares e vasculares e outras sistêmicas, incluindo manifestações orais, às quais podem atingir uma ocorrência de $80 \%$. As lesões orais mais comuns incluem: xerostomia, cárie dentária, lesões periapicais, gengivite, doença periodontal, candidíase oral, síndrome da ardência bucal, alterações no paladar, língua geográfica, líquen plano oral e estomatite aftosa recorrente. $O$ diabetes mellitus, assim como as suas complicações na cavidade oral, na maioria das vezes pode ser prevenido principalmente por meio da prática de atividades físicas, do controle da pressão arterial, dos níveis de colesterol, dos problemas emocionais, além da redução do peso corporal, enquanto, o tratamento tem como finalidade principal, a manutenção do controle metabólico e glicêmico do paciente. Considerações finais: A plena compreensão e o domínio do conhecimento da fisiopatologia, das manifestações e do próprio manejo dos diferentes tipos de infecções orofaciais relacionadas ao diabetes mellitus pelo endocrinologista e pelo cirurgião-dentista são essenciais para otimizar 0 atendimento dos pacientes diabéticos.

Palavras-chave: Boca, Diabetes mellitus, Odontologia.

\begin{abstract}
Objective: To describe oral manifestations from diabetes mellitus. Bibliographic review: Diabetes mellitus is the most common metabolic disorder, causes multivisceral repercussions, and classified as type 1 and type 2. When uncontrolled it can generate a series of renal, ocular and vascular and other systemic complications, including oral manifestations, which can reach an occurrence of $80 \%$. The most common oral lesions include: xerostomia, dental caries, periapical lesions, gingivitis, periodontal disease, oral candidiasis, burning mouth syndrome, changes in taste, geographical tongue, oral lichen planus and recurrent aphthous stomatitis. Diabetes mellitus, as well as its complications in the oral cavity, most of the time can be prevented mainly by increasing the practice of physical activities, controlling blood pressure, cholesterol levels, emotional problems, in addition to reducing the body weight, while the main purpose of treatment is to maintain the patient's metabolic and glycemic control. Final considerations: Full understanding and knowledge of the pathophysiology, manifestations and management of different types of orofacial infections related to diabetes mellitus by the endocrinologist and dentist are essential to optimize the care of diabetic patients.
\end{abstract}

Key words: Mouth, Diabetes mellitus, Dentistry.

1 Universidade Federal do Espírito Santo (UFES), Vitória - ES. *E-mail: carolthomesodonto@gmail.com

2 Faculdade Pitágoras, Imperatriz - MA.

${ }^{3}$ Faculdade Pitágoras, São Luís - MA.

${ }^{4}$ Centro Universitário Unileão, Juazeiro do Norte - CE.

${ }^{5}$ Centro Universitário ITPAC, Araguaína - TO. 


\section{RESUMEN}

Objetivo: Describir las manifestaciones bucales de la diabetes mellitus. Revisión bibliográfica: La diabetes mellitus es el trastorno metabólico más común y causa repercusiones multiviscerales, clasificadas como tipo 1 y tipo 2. Cuando no se controla puede generar una serie de complicaciones renales, oculares, vasculares y otras sistémicas, incluidas manifestaciones orales, que pueden llegar a ocurrencia del $80 \%$. Las lesiones orales más comunes incluyen: xerostomía, caries dental, lesiones periapicales, gingivitis, enfermedad periodontal, candidiasis oral, síndrome de boca ardiente, cambios en el gusto, lengua geográfica, liquen plano oral y estomatitis aftosa recurrente. La diabetes mellitus, así como sus complicaciones en la cavidad bucal, la mayoría de las veces se puede prevenir principalmente aumentando la práctica de actividades físicas, controlando la presión arterial, los niveles de colesterol, problemas emocionales, además de reducir el peso corporal, mientras que los principales El propósito del tratamiento es mantener el control metabólico y glucémico del paciente. Consideraciones finales: La comprensión y el conocimiento completo de la fisiopatología, las manifestaciones y el manejo de los diferentes tipos de infecciones orofaciales relacionadas con la diabetes mellitus por parte del endocrinólogo y el dentista son esenciales para optimizar la atención de los pacientes diabéticos.

Palabras clave: Boca, Diabetes mellitus, Odontología.

\section{INTRODUÇÃO}

O diabetes mellitus é um dos distúrbios metabólicos mais comuns e causador de repercussões multiviscerais. É caracterizado pela presença de um quadro de hiperglicemia crônica que pode ser desencadeado por deficiência de secreção de insulina, por uma resistência à ação da insulina ou pelas duas simultaneamente, podendo ser acompanhada também pela presença de alterações metabólicas referentes ao metabolismo de carboidratos, proteínas e lipídios (NAZIR MA, et al., 2018; ROHANI B, 2019; MAURIOBRADORS E, et al., 2017; TERNOIS MGM, 2017; SOUSA M, et al., 2011).

A intensidade das complicações diabéticas geralmente acompanha o grau e a duração da hiperglicemia em proporcionalidade. Quando descontrolada, pode gerar uma série de complicações renais, oculares e vasculares, sendo que os sintomas mais comumente encontrados nos portadores de diabetes mellitus incluem: quadros de polidipsia, poliúria-nictúria, polidipsia associada à xerostomia, polifagia, hálito cetônico, cãibras e emagrecimento rápido (ROHANI B, 2019; YAMASHITA JM, et al., 2013).

O número de pessoas portadoras de diabetes aumentou consideravelmente de 108 milhões em 1980 para 422 milhões em 2014. A prevalência geral de diabetes entre adultos maiores de 18 anos aumentou, portanto, de 4,7\% em 1980 para 8,5\% em 2014 e a Organização Mundial da Saúde (OMS) prevê que isso aumente até cerca de 439 milhões, quase 10\% dos adultos em 2030 (WHO, 2020).

As duas formas mais comuns de classificação da doença são o diabetes mellitus tipo I e o tipo II. Além dessas, existem outras menos frequentes, mas, que são igualmente importantes clinicamente: diabetes gestacional, diabetes associado a medicamentos; associado às doenças do pâncreas, como a fibrose cística; relacionado às infecções, como a rubéola congênita e outras relacionadas às síndromes genéticas (PRADO BN e VACCAREZZA G, 2013).

De etiologia autoimune, o diabetes mellitus tipo I, conhecido também como diabetes mellitus insulinodependente se caracteriza pela diminuição da secreção da insulina devido à destruição das células beta das ilhotas de Langherans pancreáticas que frequentemente leva à perda completa de insulina. Ocorre principalmente nos pacientes pediátricos e constitui de $5 \%$ a $10 \%$ de todos os casos de diabetes mellitus (NAZIR MA, et al., 2018; FARAG AM, et al., 2017; YAMASHITA JM, et al., 2013; PRESHAW PM e BISSETT SM, 2019).

O diabetes mellitus tipo II, tipo mais comumente encontrado $(90 \%$ dos casos de $\mathrm{DM})$ e relacionado à fatores genéticos e ambientais, é caracterizado pela resistência dos tecidos periféricos à insulina. Assim, o organismo é incapaz de utilizá-la, mesmo com a presença dela no corpo. Essa forma também é conhecida como diabetes mellitus não insulinodependente e, ocorre principalmente em adultos após os 40 anos de 
idade, sendo normalmente associada a um estilo de vida do tipo sedentário (YAMASHITA JM, et al., 2013; SOUSA M, et al., 2011; SOBRINHO KN, et al., 2014; PRESHAW PM e BISSETT SM, 2019).

Tanto o diabetes mellitus tipo I quanto o diabetes mellitus tipo II apresentam inúmeras complicações em longo prazo. Com repercussões multiviscerais, a mucosa oral e elementos dentários também constituem alvos dessa doença metabólica. As manifestações orais são dependentes do controle glicêmico do paciente e, mesmo aqueles que possuem uma boa higiene oral estão sujeitos às lesões, uma vez que a frequência de ocorrência delas pode chegar a $80 \%$. As complicações orais nesses indivíduos podem estar relacionadas à função neutrofílica deficiente, aumento de atividade da colagenase, redução da síntese de colágeno, microangiopatia e neuropatia (MAURI-OBRADORS E, et al., 2017; PRADO BN e VACCAREZZA G, 2013; ROHANI B, 2019).

A hiperglicemia prolongada, característica do DM, além de gerar alterações sistêmicas, pode alterar a função das glândulas salivares e pode causar mudanças na composição e no volume de saliva secretada (CARRAMOLINO-CUÉLLAR E, et al., 2018). Esta hipofunção da glândula salivar se deve a várias causas, como infiltração gordurosa, desidratação causada por poliúria, doença microvascular, inflamação local, irritação da cavidade bucal, distúrbios metabólicos e neuropatias, e, além disso, esta hipofunção aumenta o risco de cárie, doença periodontal, saburra lingual, halitose e candidíase oral (CARRAMOLINO-CUÉLLAR E, et al., 2018).

A hiperglicemia não controlada provoca alterações vasculares no fluxo sanguíneo e no aumento da permeabilidade com efeitos microvasculares ou macrovasculares reconhecidas nas doenças periodontais (YAMUNADEVI A, et al., 2014).

Considerando a relevância do conhecimento sobre as complicações orais do diabetes mellitus e de suas terapêuticas, o objetivo da presente pesquisa é realizar uma consulta às bases de dados PubMed e Google Scholar sobre as manifestações orais mais comuns ocasionadas pelo diabetes mellitus a fim de enfatizar a atenção dos cirurgiões-dentistas e dos médicos no diagnóstico precoce dessa doença.

\section{REVISÃO BIBLIOGRÁFICA}

\section{Diagnóstico}

O diagnóstico da diabetes mellitus para muitos pacientes têm um impacto psicossocial, ou seja, causa ansiedade, depressão, falta de energia, disfunções sexuais, dificuldades no trabalho e sensação de solidão. Então, para o paciente esse momento é importante, uma vez que marca uma nova etapa, a qual exige estilo de vida saudável, controle de peso e medicações. Entretanto, esse processo é extremamente necessário para o alcance da saúde e bem-estar do assistido; e deve ser precoce, eficaz e sustentado para prevenir complicações crônicas e evitar efeitos deletérios no metabolismo. A realização do procedimento de rastreamento do diabetes mellitus deve ser feito em todo paciente com superioridade a 45 anos de idade acerca de cada 3 anos, ou ainda mais precocemente e mais frequentemente em indivíduos assintomáticos quando os mesmos apresentarem a existência de fatores de risco para o desenvolvimento da doença. Atualmente, para realizá-lo são utilizados: glicemia plasmática em jejum; hemoglobina glicosilada; teste oral de tolerância à glicose (LAZO C e DURÁN-AGÜERO S, 2019; ADA, 2019; ASCHNER P, et al., 2016; BERTONHI LG e DIAS JCR, 2018).

A glicemia plasmática em jejum mede a glicose no sangue após, no mínimo, 8 horas de jejum. Nesse exame para o paciente ser considerado diabético o valor da glicose plasmática deve ser superior a $126 \mathrm{mg} / \mathrm{dL}$ (7.0 mmol/L). Quando comparada ao teste oral de tolerância à glicose, a glicemia plasmática em jejum é mais econômica, fácil execução, favorecendo a realização em um maior número de pessoas e apresenta um menor coeficiente de variação interindividual (ADA, 2019; BERTONHI LG e DIAS JCR, 2018).

A dosagem de hemoglobina glicosilada, também chamada de hemoglobina glicada, hemoglobina A1c ou simplesmente $\mathrm{HbA1c}$, monitora a glicose média no sangue nos últimos 2-3 meses. Para o paciente ser considerado diabético o valor de $\mathrm{A} 1 \mathrm{C}$ deve ser superior a $6,5 \%(48 \mathrm{mmol} / \mathrm{mol})$, entretanto existem alguns 
fatores que podem afetar a glicação da hemoglobina independentemente da glicemia. São eles: tratamento do HIV, idade, etnia (indivíduos asiáticos e afrodescendentes apresentam níveis mais elevados de hemoglobina glicada quando comparados à caucasianos), gravidez, genética, hemoglobinopatias, anemias hemolíticas e ferropriva (Métodos e critérios para o diagnóstico do diabetes mellitus (NAYA LD e ÁLVAREZ ED, 2016; ADA, 2019).

O teste oral de tolerância à glicose (TOTG), também conhecido como exame de curva glicêmica, determina a capacidade que um indivíduo tem de manter a homeostase da glicose sanguínea após uma sobrecarga de glicose. Para isso é realizada a medição em dois momentos: após jejum e após 2 horas da ingestão de $75 \mathrm{~g}$ de glicose dissolvida em água, segundo a OMS. Para um diagnóstico de Diabetes Mellitus a glicemia plasmática deve ser igual ou superior a $200 \mathrm{mg} / \mathrm{dL}$ após duas horas da ingestão de glicose dissolvida (NAYA LD e ÁLVAREZ ED, 2016; ADA, 2019).

\section{Prevenção}

O diabetes mellitus assim como as suas complicações manifestadas na cavidade oral, na maioria das vezes pode ser prevenido principalmente por meio do aumento da prática de atividades físicas, do controle da pressão arterial, dos níveis de colesterol, dos problemas emocionais, além da redução do peso corporal (NAZIR MA, et al., 2018).

Nos indivíduos de alto risco, ou seja, mais suscetíveis a desenvolver o diabetes mellitus que possuem uma tolerância menor à glicose ou glicemia de jejum, os aspectos preventivos também incluem mudanças em relação ao estilo de vida padrão, a prática regular de exercícios físicos, dieta adequada e até mesmo o uso de medicamentos antidiabéticos quando indicados (NAZIR MA, et al., 2018).

Tendo em vista os aspectos observados anteriormente, infere-se que as manifestações orais do diabetes mellitus podem ser minimizadas por vários aspectos preventivos que se baseiam no controle da glicemia, na prática de autocuidado em relação à doença, além da manutenção da saúde bucal de maneira consciente (NAZIR MA, et al., 2018).

Sendo assim, o envolvimento de profissionais de saúde bucal em estratégias para identificar indivíduos em risco de diabetes estenderá os esforços preventivos e de triagem necessários para retardar o desenvolvimento dessas doenças (LEITE RS, et al., 2013).

\section{Tratamento}

O tratamento do diabetes mellitus tem como finalidade envolver principalmente a manutenção do controle metabólico e glicêmico do paciente. O diabetes mellitus é uma doença de caráter metabólico incurável, no entanto, pode ser controlada (BERTONHI e DIAS, 2018; COSTA AC, 2018).

Dentro desse contexto, é estritamente necessário que o médico oriente o paciente a seguir tanto as mudanças aplicadas em seu estilo de vida que se referem principalmente a prática regular de exercícios físicos e o seguimento de dieta específica quanto à prescrição de medicamentos (VILLAS BOAS LCG, et al., 2012).

A terapêutica medicamentosa do diabetes mellitus se baseia geralmente no uso de antidiabéticos orais e na insulinoterapia. O uso da metformina geralmente é recomendado para o tratamento inicial com medicamentos. Se a metformina for contraindicada, mal tolerada ou inadequadamente eficaz, inúmeras alternativas terapêuticas e suplementos encontram-se disponíveis (PFEIFFER AFH e KLEIN HH, 2014).

Grande parte dos ensaios clínicos sobre o assunto disponíveis demonstraram que as sulfonilureias e a insulina são benéficas em relação aos parâmetros de avaliação relevantes para o paciente, mas dados comparáveis de ensaios clínicos ainda não estão disponíveis para qualquer outro medicamento antidiabético (exceto metformina) (PFEIFFER AFH e KLEIN HH, 2014).

A terapêutica deve ser orientada de forma individual, tendo em vista a idade do paciente, o estágio da doença, peso corporal, comorbidades, situação de trabalho, adesão e prioridades pessoais. Portanto, a combinação além de dois antidiabéticos não é recomendada de acordo com esses parâmetros (PFEIFFER AFH e KLEIN HH, 2014). 


\section{Manifestações orais do diabetes mellitus}

As manifestações orais provenientes do quadro do diabetes mellitus são comuns e podem afetar tanto o controle metabólico da doença quanto o tratamento odontológico. É de extrema importância que os profissionais da área da saúde atuem no controle metabólico dos portadores dessa doença. As principais manifestações orais e complicações relacionadas ao quadro de diabetes mellitus incluem xerostomia, cárie dentária, lesões periapicais, doença periodontal, queimação na boca e alterações no paladar (MAURIOBRADORS E, et al., 2017; PRADO BN e VACCAREZZA G, 2013; ROHANI B, 2019).

\section{Xerostomia}

Os pacientes portadores de diabetes mellitus geralmente apresentam disfunções relacionadas à saliva que podem reduzir o fluxo salivar, além de alterações relacionadas às mudanças na composição salivar. Sendo assim, a prevalência universal estimada de xerostomia em pacientes portadores da doença varia entre $34 \%$ e $51 \%$ (ROSAS CY, et al., 2018; ROHANI B, 2019).

O quadro de xerostomia pode ocasionar inúmeros problemas, como dificuldades na alimentação, no ato de engolir e até no falar, causando um impacto negativo significativo na qualidade de vida dos pacientes com diabetes mellitus. A etiologia dessa condição ainda é desconhecida, mas, pode se relacionar a poliúria, neuropatias autonômicas, alterações microvasculares e alterações nas membranas basais das glândulas salivares. Além disso, existe uma inter-relação significativa entre o grau de xerostomia e os níveis de glicose na saliva. Portanto, o nível mais alto de disfunção salivar geralmente é notado em diabéticos com pobre controle glicêmico (ROSAS CY, et al., 2018; ROHANI B, 2019).

O tratamento da xerostomia deve-se evitar fatores irritantes como, a ingestão de grande quantidade de água e líquidos sem açúcar, uma higienização ideal com aplicação regular de flúor que busque auxiliar no processo de remineralização do esmalte. Além disso, outros tratamentos auxiliares são representados pela estimulação salivar natural e artificial, o uso de salivas artificiais, géis e pastilhas lubrificantes, spray de mucina, além do próprio uso de ômegas que diminuem os sintomas, uso de corticosteroides orais e até mesmo a irrigação das glândulas parótidas com medicamentos. Dessa forma, medicamentos sialagogos podem colaborar com o estímulo do fluxo salivar como, por exemplo a pilocarpina e a cevimelina, o uso de antiinflamatórios para tratamento de mialgias quando necessário. Por último, regularmente deve-se realizar consultas ao cirurgião-dentista para acompanhamento (ADIVÍNCULA MA e PIEDADE SF, 2016).

\section{Cárie dentária}

Pacientes diabéticos encontram-se mais suscetíveis ao desenvolvimento de cáries dentárias novas e recorrentes. Essa incidência alta deve-se em grande parte pela redução da capacidade de limpeza e tamponamento da saliva, aumento de carboidratos presentes na saliva, além do aumento de leveduras orais, Streptococcus mutans e Lactobacilos. Além disso, o quadro hiperglicêmico crônico pode levar a pulpites crônicas irreversíveis, podendo progredir para casos de necroses pulpares (ROHANI B, 2019).

A terapêutica da cárie envolve principalmente a aplicação de programas de prevenção individualizada representada pela adição de mais consultas de controle dentário, aplicações tópicas de flúor em gel, educação de higiene oral com ênfase na importância de uma higiene oral meticulosa. Além do próprio uso de flúor em forma de bochechos ou em aplicações periódicas no cirurgião-dentista também colaboram com a prevenção da cárie (CARVALHO DFN, 2013).

A definição da presença de lesão de cárie, do diagnóstico e da terapêutica empregada de acordo com cada caso envolve a utilização de diferentes métodos de diagnóstico, desde o exame clínico (inspeção visual e exame tátil) e o exame radiográfico, até métodos mais complexos de ordem complementar como o uso do ultrassom, corante e o uso laser (CARVALHO DFN, 2013).

\section{Lesões periapicais}

Os pacientes diabéticos com o quadro da doença não controlada, apresentam maior prevalência de lesão periapical. Afirma-se que, se a hiperglicemia não estiver controlada, a cicatrização da lesão periapical não irá ocorrer e esta irá aumentar, mesmo com o tratamento endodôntico. Assim, a polpa que acaba não possuindo 
a circulação limitada irá causar comprometimentos na resposta imune, aumentando o risco destes à infecção ou necrose pulpar (FERREIRA CM, et al., 2014; MAURI-OBRADORS E, et al., 2017).

As lesões periapicais são caracterizadas como doenças infecciosas desencadeadas por micro-organismos que infectam o sistema de canais radiculares. Nos casos de necropulpectomia, o profissional endodontista lida com o combate do quadro de infecção primária do canal, enquanto que nos casos de retratamento endodôntico estão relacionados a existência de uma infecção persistente ou secundária do canal (SIQUEIRA JR JF, et al., 2012).

De maneira conceitual, as lesões periapicais são tratadas por meio da eliminação dos micro-organismos. É neste sentido que se insere justamente o tratamento de elementos dentários com polpa necrosada, ou seja, além da importância da prevenção da introdução de novos micro-organismos no interior do sistema de canais radiculares, deve-se ter como base prioritária eliminar a infecção endodôntica ou até mesmo reduzi-la de forma significativa para que se tenha êxito nessa terapêutica (SIQUEIRA JR JF, et al., 2012).

\section{Doença periodontal}

A deficiência do controle glicêmico pode estar associada ao aparecimento e a progressão do quadro de gengivite e periodontite. Nesse sentido, a doença periodontal tem tido uma maior prevalência e incidência em pacientes portadores de diabetes mellitus tipo I e tipo II e com prevalência grave em pacientes diabéticos (59\%) em comparação com os pacientes não diabéticos (39\%). Dentro desse contexto, a doença periodontal expressa um impacto negativo sobre o diabetes, assim como o controle da doença periodontal exerce efeitos desejáveis em relação ao controle da glicemia. A própria eliminação de patógenos que ocorre na terapêutica periodontal leva a uma redução no quadro de inflamação, corroborando com a diminuição da resistência à insulina e assim, reduzindo os níveis de glicose (INDURKAR MS, et al., 2016; KHAN T, 2018; DANIEL R, et al., 2012; ROHANI B, 2019).

O tratamento da doença periodontal traz inúmeros benefícios para o controle glicêmico do diabetes, o que torna o profissional periodontista uma peça importante durante o acompanhamento do paciente diabético. Dentro desse contexto, os pacientes diabéticos devem ser orientados em relação ao risco do diabetes para a doença periodontal. Além disso, os mesmos devem ser encorajados a possuírem uma higiene oral criteriosa e a entenderem que o controle de placa bacteriana quando praticado em casa é a maneira na qual o quadro de inflamação que conduz a destruição do periodonto será mantida sob controle. Ademais, esses pacientes devem receber uma terapia periodontal de suporte com base em intervalos necessários para manter um alto nível de saúde periodontal e, além disso, a frequência das consultas deve ser definida pelo periodontista considerando a extensão e o grau de severidade da doença periodontal, com a habilidade do paciente para a prática dos procedimentos de higiene oral e com o nível do controle metabólico do paciente (ABREU IS, et al., 2014).

\section{Queimação na boca}

A sensação de ardência na boca ou disestesia na cavidade oral de pacientes diabéticos se atribui ao próprio controle glicêmico ineficaz, alterações no metabolismo da mucosa oral, angiopatia, candidíase e neuropatia. Nesses pacientes, a dor neuropática pode se manifestar por meio de queimação, formigamentos ou até mesmo choque elétrico ou sensação de pontada que têm efeitos significativos nas próprias funções físicas e psicológicas associadas à distúrbios do sono, depressão e quadros de ansiedade (ROHANI B, 2019).

Os tratamentos disponíveis se baseiam no uso tópico de medicamentos à base de Clonazepam, medicamentos dos tipos sistêmicos com ácido alfa-lipóico, inibidor seletivo da recaptação da serotonina e amisulprida, e por meio de terapia comportamental cognitiva. Além disso, outras alternativas conceituadas com base na opinião de experts e na prática clínica, mas não ainda avaliadas de forma concreta, incluem os tratamentos também tópicos à base de medicamentos como capsaicina, doxepina e lidocaína, além de tratamentos de ordem sistêmica tais como o uso de medicamentos antidepressivos tricíclicos, inibidores da recaptação de norepinefrina serotonina, anticonvulsivantes, opioides e benzodiazepínicos como Clonazepam e Alprazolam (MONTADON AAB, et al., 2011). 


\section{Alterações no paladar}

A presença de disfunções relacionadas ao paladar pode atingir pacientes diabéticos sem o controle da doença, podendo ocasionar alterações da sensação do paladar ou até mesmo aumento dos limites de detecção por meio de quadros de neuropatia, sendo que essa alteração sensorial pode causar a inibição da capacidade de manter uma boa dieta, levando a uma regulação deficiente da glicose (ROHANI B, 2019).

A predominância de um controle rigoroso da glicemia demonstra ser fundamental no processo da estabilização e, mesmo, para a melhora das alterações relacionadas a neuropatia diabética correlacionada as alterações no paladar. Nesse sentido, todo empenho possível deve ser aplicado para que o paciente seja mantido em um estado característico de normoglicemia (NASCIMENTO OJM, et al., 2016).

Inúmeras evidências inferem que o quadro de estresse oxidativo se encontra envolvido com os princípios das alterações relacionadas a neuropatia diabética. Dessa forma, o uso de fármacos antioxidantes seria uma ótima alternativa terapêutica. O ácido $\alpha$-lipoico administrado por via venosa é, atualmente, o único tratamento baseado no mecanismo da doença com eficácia comprovada e passível de utilização na prática clínica. A utilização do mesmo medicamento por via oral, que representa a única apresentação atualmente disponível no Brasil, ainda precisa de mais estudos comprobatórios, por mais que os indícios sugiram sua eficácia. (NASCIMENTO OJM, et al., 2016).

\section{CONSIDERAÇÕES FINAIS}

O diabetes mellitus é uma doença que não apresenta cura, e seu controle se dá por meio de atividades físicas habituais, dieta regulada e uso dos medicamentos antidiabéticos. As evidências apontam que complicações orais crônicas e persistentes nesses pacientes afetam adversamente a glicemia. Neste sentido, a prevenção e o controle das complicações orais são consideráveis. Portanto, médicos e cirurgiões-dentistas devem estar atentos às diversas manifestações orais do diabetes com o intuito de realizar o diagnóstico precoce.

\section{REFERÊNCIAS}

1. ABREU IS et al. Diabetes Melittus: O que os periodontistas devem saber. Brazilian Journal of Periodontology, 2014; $24(4): 22-28$.

2. AMERICAN DIABETES ASSOCIATION (ADA). Classification and diagnosis of diabetes: standards of medical care in diabetes-2019. Diabetes Care, 2019; 42 (1): 13-28.

3. ASCHNER P, et al. Guía de práctica clínica para la prevención, diagnóstico, tratamiento y seguimiento de la diabetes mellitus tipo 2 en la población mayor de 18 años. Colombia Médica, 2016; 47(2): 109-30.

4. BERTONHI LG, DIAS JCR. Diabetes mellitus tipo 2: aspectos clínicos, tratamento e conduta dietoterápica. Revista Ciências Nutricionais, 2018; 2(2): 1-10.

5. CARRAMOLINO-CUÉLLAR, E et al. Salivary flow and xerostomia in patients with type 2 diabetes. Journal of Oral Pathology \& Medicine, 2018; 47, (5): 526-530.

6. CARVALHO DFN. Diabetes melittus e cárie dentária. Dissertação (Mestrado em Medicina Dentária) - Universidade Católica Portuguesa, Viseu, 2013; 83p.

7. COSTA AC. Manifestações da Diabetes Mellitus na cavidade oral. Dissertação (Mestrado em Medicina Dentária) Universidade Fernando Pessoa, Porto, 2018; 26 p.

8. DANIEL R, et al. Diabetes and periodontal disease. Journal of Pharmacy \& Bioallied Sciences, 2012; 4(2): $280-282$.

9. FARAG AM, et al. Head and Neck Manifestations of Endocrine Disorders. Atlas of the oral and Maxillofacial Surgery Clinics, 2017; 25(2): 197-207.

10. FERREIRA, CM et al. Prevalência de lesão endodôntica em pacientes diabéticos. Revista Brasileira de Promoção em Saúde, 2014; 27 (2): 163 - 168.

11. INDURKAR MS, et al. Oral manifestations of diabetes. American Diabetes Association, $2016 ; 34$ (1) : $54-57$.

12. KHAN T. Oral manifestations and complications of diabetes mellitus: A review. International Journal of Medical Health, 2018; 4: $50-52$.

13. LAZO C, DURÁN-AGÜERO S. Efecto del diagnóstico de la diabetes mellitus y su complicación con los trastornos de la conducta alimentaria. Revista Chilena de Nutrición, Santiago, 2019; 46 (3): 352-360.

14. LEITE RS, et al. Oral health and type 2 diabetes. The American Journal of the Medical Sciences, $2013 ; 345$ (4) :271 -273 .

15. MAURI-OBRADORS E, et al. Oral Manifestations of Diabetes mellitus. A systematic review. Medicina Oral Patologia Oral y Cirurgia Bucal, 2017; 22 (5) :586 - 594. 
16. MENDES EV. O cuidado das condições crônicas na atenção primária à saúde: o imperativo da consolidação da estratégia da saúde da família [Internet]. Brasília: OPAS; 2012. 512 p.

17. MONTADON AAB, et al. Síndrome da ardência bucal: avaliação e tratamento. Revista de Odontologia da Universidade Cidade de São Paulo, 2011; 23 (1): 59 - 69.

18. NASCIMENTO OJM, et al. Neuropatia diabética. Revista Dor, 2016; 17 (1): $46-51$.

19. NAYA LD, ÁLVAREZ ED. Diabetes mellitus. Criterios diagnósticos y clasificación. Epidemiología. Etiopatogenia. Evaluación inicial del paciente con diabetes. Medicine-Programa de Formación Médica Continuada Acreditado, 2016; 12 (17): 935-946.

20. NAZIR MA, et al. The burden of Diabetes, its oral complications and their prevention and management. Journal of Medical Sciences, 2018; 6 (8) :1545 - 1553.

21. PFEIFFER AFH, KLEIN HH. The treatment of Type 2 Diabetes. Deutsches Ärzteblatt International, v. $2014 ; 111$ (5): $69-82$.

22. PRADO BN, VACCAREZZA G. Alterações bucais em pacientes diabéticos. Revista de Odontologia da Universidade Cidade de São Paulo, 2017; 25 (2) :147.

23. PRESHAW PM, BISSETT SM. Periodontitis and diabetes. British Dental Journal, $2019 ; 227$ (7) : 577-584.

24. ROHANI B. Oral Manifestations in patients with diabetes mellitus. World Journal of Diabetes, $2019 ; 10$ (9): 485 - 489.

25. ROSAS CY, et al. Dental, periodontal and salivary conditions in diabetic children associated with metabolic control variables and nutritional plan adherence. European Journal of Paedratic Dentistry, 2018;19 (2) :119 - 126.

26. SIQUEIRA JR JF, et al. Princípios biológicos do tratamento endodôntico de dentes com polpa necrosada e lesão perirradicular. Revista Brasileira de Odontologia, 2012; 69 (1) : 8- 14.

27. SOBRINHO KN, et al. Alterações em mucosa bucal de pacientes portadores de diabetes mellitus. Revista de Odontologia da Universidade Cidade de São Paulo, 2017; 26 (3): 200- 204.

28. SOUSA MGM, et al. Clinical study of the oral Manifestations and Related factors in type 2 diabetics patients. Brazilian Journal of Otorhinolaryngology, 2011;77 (2) :145-152.

29. TERNOIS M. La bouche: un miroir du diabète. La Presse Médicale, 2017; 46 (9): 822-830.

30. VILLAS BOAS LCG, et al. Relação entre apoio, adesão aos tratamentos e controle metabólico de pessoas com diabetes mellitus. Revista Latino-Americana de Enfermagem, 2012;20 (1) :1-8.

31. WORLD HEALTH ORGANIZATION (WHO). Painel do WHO Coronavirus Disease (COVID-19) Dashboard. 2020. Disponível em: https://covid19.who.int/.

32. YAMASHITA JM, et al. Manifestações bucais em pacientes portadores de Diabetes Mellitus: uma revisão sistemática. Revista de Odontologia da UNESP, 2013;42 (3): 211-220.

33. YAMUNADEVI A. et al. Morphological alterations in the dentition of type I diabetes mellitus patients. Journal of pharmacy \& bioallied sciences, 2014; 6 (1). 